\title{
Electrical measurement of the linewidth of a quantum well bound state
}

\author{
Z. A. Kobos ${ }^{\mathrm{a}, *}$, A. Noonan ${ }^{\mathrm{a}}$, M. A. Reed ${ }^{\mathrm{a}, \mathrm{b}}$ \\ ${ }^{a}$ Department of Electrical Engineering, Yale University, New Haven, CT 06520, USA \\ ${ }^{b}$ Department of Applied Physics, Yale University, New Haven, CT 06520, USA
}

\begin{abstract}
We investigate electron tunneling spectroscopy in the presence of a bound state within a double quantum barrier, single quantum well structure. We demonstrate a new technique to directly measure the intrinsic linewidth of the bound state within the quantum well from the current-voltage signature of the resonant tunneling phenomena and contrast our results with the standing approach in the literature. We then examine the signal behavior for the influence of device temperature and find support for electron-electron interactions within the well. The measured intrinsic bound-state width, $\Gamma_{E}$, in the negative differential conductance regime is $1.11 \pm 0.01 \mathrm{meV}$.
\end{abstract}

Keywords: quantum well, resonant tunneling diode, tunneling spectroscopy

PACS: 72.10.-d, 73.21.Fg, 73.40.Gk, 73.40.Kp, 73.63.Hs

\section{Introduction}

\subsection{Motivation}

In the transition from the macroscopic to the mesoscopic, changes in electron dimensionality have given rise to exotic device physics exploiting electron confinement to modify the transport physics. Researchers made use of tunneling spectroscopy into and through bound states for a variety of different device geometries: 3D-0D-3D [1, 2], to and from twodimensional electron gas (2DEG) into 2D and 0D states[3-5], as well as 3D-2D-3D processes in semiconductor heterostructures 6

Tunneling via an intermediary bound state present in the system gives rise to a peak in conductance [1, 2] when incoming electrons have energies corresponding to that of the bound state. The work of Tsuchiya, et al.[6], established an analogous signature in the second derivative of the current-voltage characteristic of the tunneling current for a double quantum barrier, single quantum well (DQB) system. Because this signature occurs at the onset of appreciable current passing through the device (corresponding to minimal accumulated charge density within the well region), this technique claims to measure the intrinsic properties of the bound state within the quantum well, a notion that has propagated throughout the literature [7-9].

We propose and subsequently demonstrate a new application of tunneling spectroscopy to measure the bound state parameters in the negative differential conductance regime of the DQB structure, and show that these parameters are markedly different from those extracted via the existing approach. Our technique should prove useful for researchers seeking non-destruc characterization of double quantum barrier (also known as resonant tunneling diode) structures for terahertz oscillator applications [

\footnotetext{
${ }^{*}$ Corresponding author

Email address: zachary.kobos@yale.edu (Z. A. Kobos)
}

\section{Materials \& Methods}

To investigate an idealized case of resonant tunneling through a bound state, a previously-characterized[11] double-barrier GaAs$\mathrm{AlGaAs}$ resonant tunneling diode was studied.

Current-voltage measurements were taken with a HP 4156B semiconductor parameter analyzer. Harmonic detection of derivatives of the current was accomplished with two SRS830 lock-in amplifiers monitoring the first and second harmonics, respectively. A Yokogawa 7651 voltage source and Agilent 33210A function generator provided the DC bias and excitation signal, respectively, to custom-made voltage summer circuitry before being supplied to the device. The bias voltage was increased in a step-wise manner for all device scans with a DC ramp rate never exceeding $0.5 \mathrm{mV} / \mathrm{s}$.

All measurements were conducted with the sample positioned inside a Janis SVT-100 continuous vapor flow cryostat. Sample temperature was controlled by flowing liquid helium through a heater-wound capillary. Activation of the PID-controlled heater resulted in warm helium vapor flowing over the device.

\section{Theory}

\subsection{Tunneling spectroscopy}

Here we utilize tunneling current measurements to directly examine the electronic properties of a bound state, engineered in a classic double quantum barrier, single quantum well system, as shown in Fig. 1. a). The conduction band of the doublebearrier heterostructure forms a confining potential, producing the quantum well bound state. The energy of conduction elec109 . shown in Fig. 1 b), resulting in resonant tunneling through the bound state[12].

The onset of resonant tunneling produces a rise in device current followed by a steep drop as the applied bias pushes the 

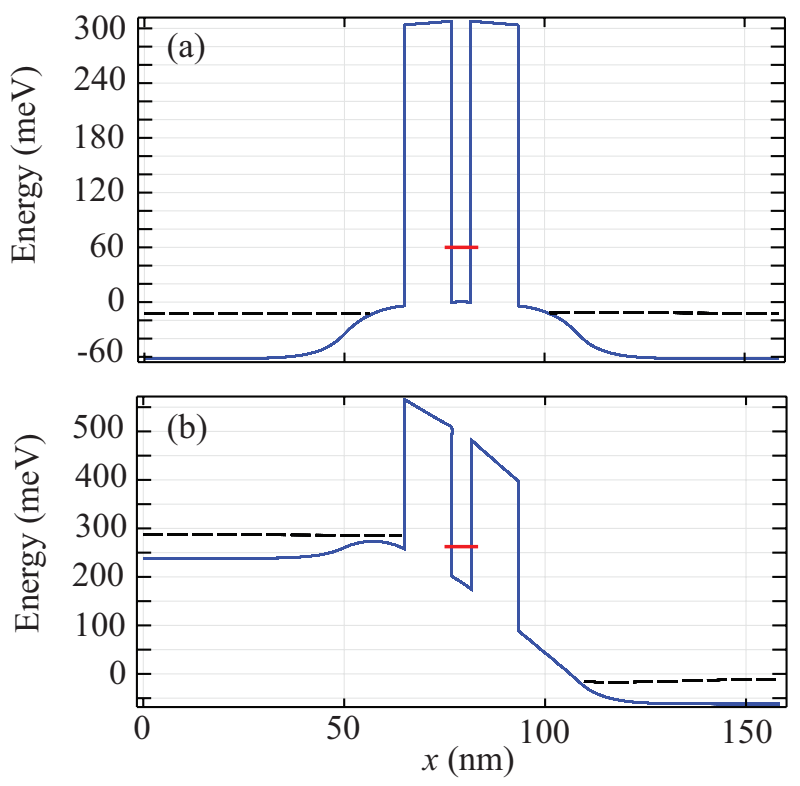

Figure 1: COMSOL-generated plots of the self-consistent band diagrams (blue) and quasi-equilibrium Fermi energies (black, dashed) for the device under test (a) at equilibrium and (b) biased to the point of shut-off for resonant tunneling. The lowest-energy bound state is illustrated in red.

bottom of the conduction band beyond the resonant state, as seen in Fig. 2(a). To elucidate the shut-off of resonant tunneling, we also plot the conductance, $G$ (Fig. 2(b)), and $d G / d V$ (Fig. 2(c)). We observe that the $d G / d V$ signal gives a sharp measurement of the shut-off width. Further on, we will compare how this feature corresponds to the quantum well bound state lifetime, and compare that to the linewidth derived from the resonant activation previously investigated in the literature [6].

Modelling the tunneling barrier as a scattering region, the current flowing through the device depends upon the energy distribution of incoming electrons in the source (the supply function), the transmission function of the active region of the device, and the availability of states in the drain into which electrons can tunnel[13, 14]:

$$
J=\frac{e}{h} \int_{-\infty}^{\infty} T(E)\left[f_{L}\left(E-\mu_{L}\right)-f_{R}\left(E-\mu_{R}\right)\right] d E
$$

where $\mathrm{T}(\mathrm{E})$ is the transmission function of the scattering region and $f_{L}$ and $f_{R}$ are the Fermi distribution functions of the left and right leads with chemical potentials $\mu_{L}$ and $\mu_{R}$, respectively. Incorporating the influence of bias voltage yields[15]:

$$
\begin{aligned}
J(V)=\frac{e m^{*} k_{B} T}{2 \pi^{2} \hbar^{3}} \quad & \int_{e V}^{\infty} \frac{\left(\frac{1}{2} \Gamma_{E}\right)^{2}}{\left[E-\left(E_{0}-\alpha e V\right)\right]^{2}+\left(\frac{1}{2} \Gamma_{E}\right)^{2}} \\
& \times \ln \left(\frac{1+\exp \left(\frac{\mu+e V-E}{k_{B} T}\right)}{1+\exp \left(\frac{\mu-E}{k_{B} T}\right)}\right) d E
\end{aligned}
$$

where $\mathrm{E}_{0}$ is the energy level of the quantum well bound state, of width $\Gamma_{E}$, with respect to the bottom of the well, $\mu$ specifies the chemical potential with respect to the bottom of the
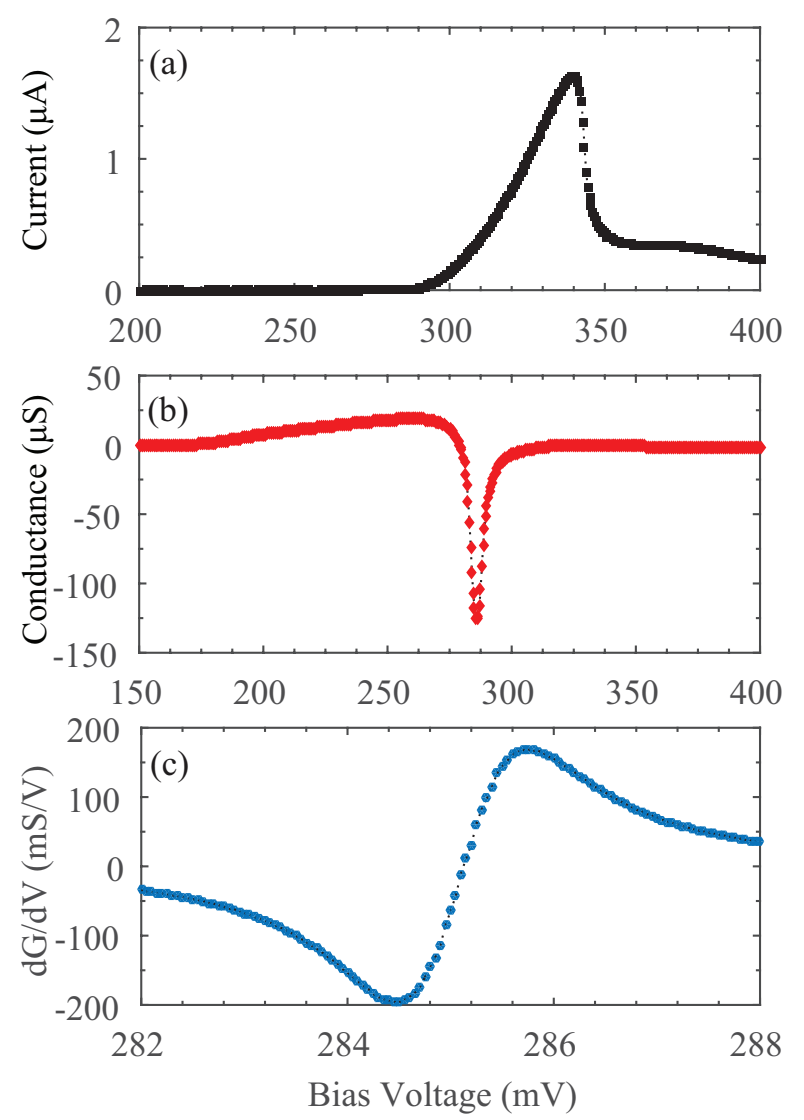

Figure 2: Device characterization showing signatures of resonant tunneling in the (a) current, (b) conductance (acquired at $1.8 \mathrm{mV}_{r m s}$ ), and (c) $d G / d V$ profiles $\left(197 \mu \mathrm{V}_{r m s}\right)$ of the device under investigation. All measurements are taken at $4.2 \mathrm{~K}$.

conduction band in the degenerately-doped source and drain, and $\alpha$ is the proportion of bias voltage dropping across the second barrier ( $\frac{1}{2}$ for fully symmetric barriers) and governs the relationship between the energy width and voltage width of the feature, $\Gamma_{E}=\alpha \Gamma_{V}$. The first term inside the integral is $T(E, V)$ and the second is the combined supply functions of the of the source and drain after integrating over $\overrightarrow{k_{\|}}$.

Following the analysis and results of Tsuchiya, et al.[6], it is possible to measure the electrical properties of a bound state in a double-quantum barrier structure from features in the second derivative of $J(V)$. The density of states in the source decreases linearly with energy before transitioning to exponential decay above the Fermi energy of the lead. This kink in the supply function becomes a peak at the Fermi energy when twice-differentiated with respect to energy, $\delta\left(E-E_{F}\right)$ in the zero-temperature limit. Inserting this result into Eqn. 2, the second derivative of the current-voltage characteristic should be proportional to the transmission function of the double-barrier structure at the onset of resonant tunneling, when the Fermi energy in the source aligns with the bound state in the well. This result holds if the bound state lies well above the Fermi level in the leads $\left(E_{0}(V)-\mu \gg \Gamma_{E}\right)$ at equilibrium and is broader than the thermal energy and measurement resolution, and there must be a sizeable population of incident electrons available to 

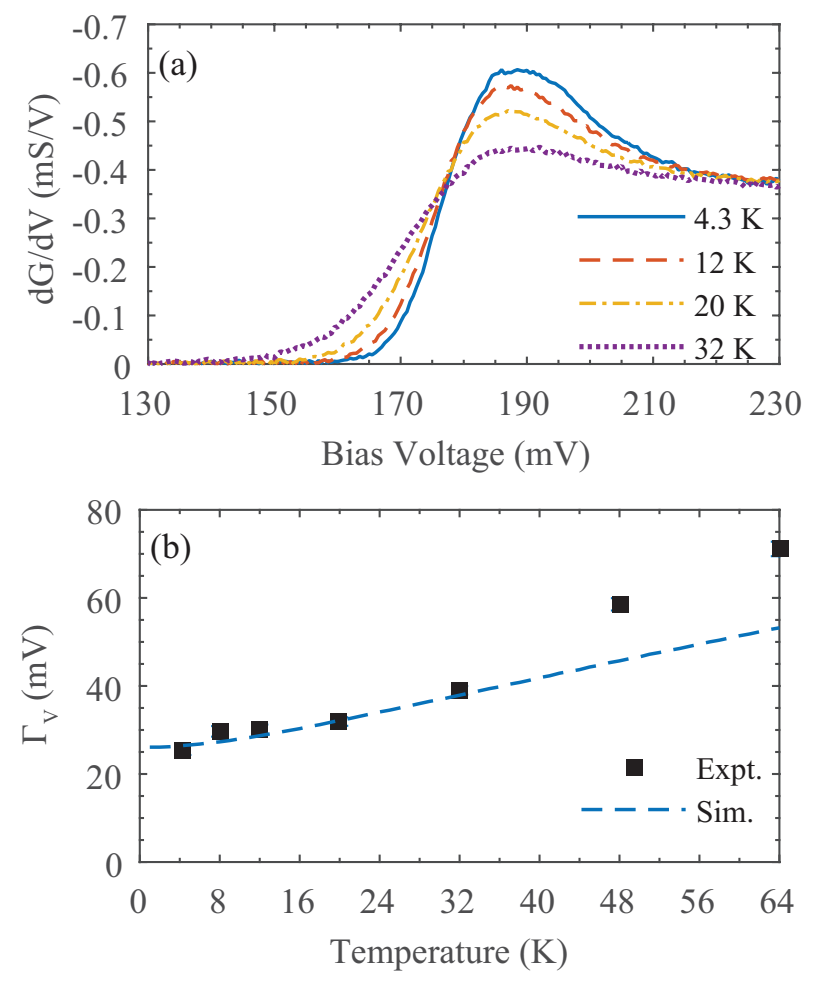

Figure 3: (a) Observed resonance activation signature in $d G / d V$ at various temperatures (solid line, $4.3 \mathrm{~K}$; dashed line, $12 \mathrm{~K}$; dot-dash, $20 \mathrm{~K}$; dotted, $32 \mathrm{~K}$ ). (b) Experimental feature width (fit to the rising edge of the signal in $a$ )) and simulated values, using $\Gamma_{E}=13 \mathrm{meV}$, for the feature's FWHM as a function of temperature. Errors from fit, and are smaller than marker size.

participate in tunneling.

\section{Results \& Discussion}

The onset of resonance activation for various temperatures is shown in Fig. 3 a). At low temperatures we observe the expected peak structure, however, this peak is superimposed upon a background plateau, corresponding to a linearly-increasing conductance with applied bias. Performing peak fitting on the rising edge of the resonance activation signature, we extract the thermal dependence of the feature width, shown in Fig. 3 (b). From the intercept, we should observe the intrinsic linewidth of the bound state. We find $\Gamma_{V} \approx 25 \mathrm{mV}$, and simulation results using $\Gamma_{E} \approx 13 \mathrm{meV}$ reasonably reproduce the observed behavior for temperatures below $32 \mathrm{~K}$. Our linewidths agree reasonably well with Tsuchiya's work, $13 \mathrm{meV}$ v. $15 \mathrm{meV}$.

We satisfy the criteria necessary for the resonance activation results to hold: calculations for our device predict the bound state to lie $60 \mathrm{meV}$ above the Fermi level in the leads. Depending upon the bias magnitude, the computed Fermi level in the source ranges from 25 to $50 \mathrm{meV}$ above the conduction band at the space-charge region/double quantum barrier interface. Both the thermal energy $(317 \mu \mathrm{eV}$ at $4.3 \mathrm{~K})$ and measurement resolution $(50-100 \mu \mathrm{V})$ fall below measured values for $\Gamma_{E}$. Yet the presence of the plateau region decreases confidence in extracted parameters from the Tsuchiya, et al, approach. We suspect this deviation from a simple Lorentzian signature arises from complications in the transport physics due to band bending in the space-charge region that occurs around the onset of resonance.

Referring back to Fig. 2(c), we observe features in $d G / d V$ around the peak-to-valley transition in the current-voltage profile nearly an order of magnitude narrower than the resonance activation signature. We now turn our attention to the origins and explanation of this much narrower feature.

The resonant contribution to tunneling current shuts off in the peak-to-valley transition of the current-voltage profile. Here there is a difference in the density of states involved as opposed to the resonance activation regime. Here, the distinguishing feature is not a kink in the supply function at the Fermi level but a Heaviside step function at the bottom of the conduction band, convoluted with the transmission function and electronic density of states within the integrand. Normally the step function defines the lower limit of integration of Eqn. 2 when modeling device behavior. Incorporating it explicitly and differentiating once with respect to voltage, we find that the differential conductance of the device should reflect the integrand of Eqn. 2 evaluated at the DC bias.

The derivative of conductance, $d G / d V$, correspondingly reflects the first derivative of the Lorentzian transmission function, whose peak-to-peak $(p)$ distance is a direct measure of the intrinsic linewidth of the bound state, $p=\Gamma_{V} / \sqrt{3}$. The peak-topeak feature width in $d G / d V$ offers a much more precise measure of $\Gamma_{V}$ than curve fitting of data in the turn-on region.

To find the intrinsic properties of the bound state of the device, we investigate the thermal dependence of the feature width at a fixed and small modulation voltage. We recorded device $d G / d V$ at multiple temperatures using $197 \mu V_{r m s}$ modulation voltage so chosen as to minimize the influence of any modulation broadening. Empirical justification of this decision is provided in the supplementary information. Measurements were taken for temperatures ranging from $4.3 \mathrm{~K}$ to $64 \mathrm{~K}$, as shown in Fig. 4 a). The thermal energy in the sample becomes comparable to $\Gamma_{E}$ at $\sim 9.5 \mathrm{~K}$, at which point we begin to see deviations from low-temperature behavior in Fig. 4(b). The feature width exhibits a parabolic dependence upon temperature, in conjunction with decreasing amplitude. The zero-temperature intercept of the parabolic fit for the temperature-broadening data (Fig. 4) should reflect the intrinsic linewidth of the bound state. Using the relationship between feature width and the bound state linewidth as well as the aforementioned voltage-to-energy conversions, we extract the energy-space linewidth $\left(\Gamma_{E}\right)$ of the quantum well bound state, finding it to be $1.11 \pm 0.01 \mathrm{meV}$.

In Fig. 1.(b), wherein the non-trivial transmission function selectively filters the population of electrons engaged in the tunneling process, and since resonant tunneling is an elastic process with a signature typically occurring with $e V \gg k_{B} T$, the occupancy in the drain is negligible. We expect an approximately constant DOS near the conduction band minima of the source arising from the degenerate doping. Therefore, we expect no thermal dependence in the simulation, since $\Gamma_{E}$ is not explicitly a function of temperature.

However, this does not exclude the possibility of a temperaturedependent carrier lifetime. Past research[16] involving tunnel- 

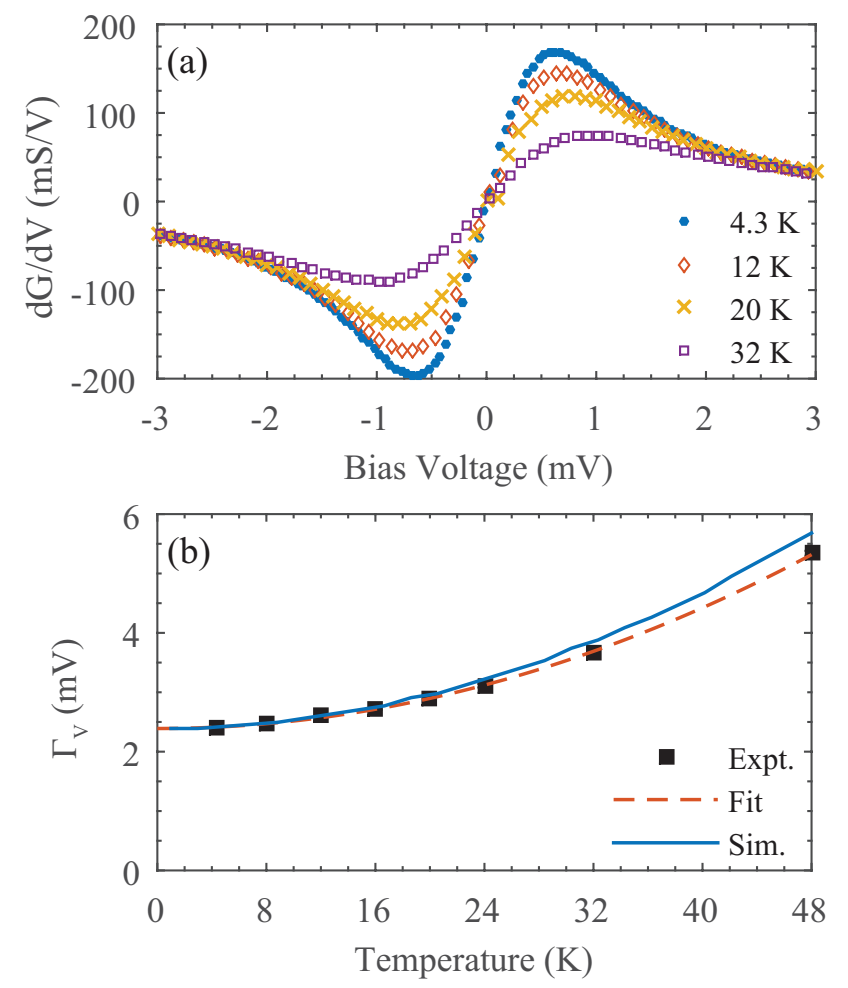

Figure 4: (a) Experimental results for the temperature-broadening of the resonant tunneling feature (closed circle, $4.3 \mathrm{~K}$; open diamond, $12 \mathrm{~K}$; cross, $20 \mathrm{~K}$; open square, $32 \mathrm{~K}$ ). The zero-crossing of each scan is set at a bias voltage of zero millivolts. (b) Feature width in $d G / d V$ as a function of temperature (black squares). A fit to the data (dashed orange line) gives a parabolic dependence of $\left(13.08 k_{B} T\right)^{2}$. The prediction of the simple model (Eqn. 2] exhibits no thermal dependence until the influence of electron-electron scattering on the linewidth is added (solid blue line). $\Gamma_{V}$ in the zero-temperature limit, from the parabolic fit, is $2.39 \mathrm{mV}$.

ing between 2DEGs has demonstrated that the parabolic dependence of the linewidth is due to electron-electron scattering within the 2DEG:

$$
\begin{aligned}
\frac{\hbar}{\tau_{e-e}}= & \frac{E_{F}}{2 \pi}\left(\frac{k_{B} T}{E_{F}}\right)^{2} \\
& \times\left[\ln \left(\frac{E_{F}}{k_{B} T}\right)+\ln \left(\frac{2 q_{T F}}{k_{F}}\right)+1\right]
\end{aligned}
$$

where $\tau_{e-e}$ is the scattering lifetime, $E_{F}$ the Fermi level within the well, and $q_{T F}$ the 2D Thomas-Fermi wave vector. The decrease in carrier life-time due to electron-electron scattering is not considered in our simple model. Incorporating the effect of electron-electron scattering via Eqn. 3 into the model's line-width parameter (as shown in Fig. 4.b)) brings simulation results in close agreement with experimental data.

\section{Conclusion}

The onset of resonant tunneling, as previously investigated, gives $\Gamma_{E} \approx 13 \mathrm{meV}$, in line with past results. Turning our attention to the signature arising from the shut-off of resonant tunneling, we find $\Gamma_{E}=1.11 \pm 0.01 \mathrm{meV}$, consistent with experimental tests of thermal broadening[16]. Our interpretation of results suggests that measurements of the bound state properties in the turn-on region do not reflect bound state parameters near or in the negative differential resistance regime. In the literature, the deviation between ab initio calculations and experimentallydetermined linewidths has been attributed to monolayer thickness fluctuations in the quantum well or barrier width. We would expect such fluctuations to effect the activation and shutoff signatures similarly: either this is not the case, or some other phenomenon determines the minimum line-width of the activation signature. One possible explanation for the difference between the two measurement regimes is the influence of longrange scattering between ionized impurities with carriers in the well[16, 17]. At higher current densities there are correspondingly higher carrier densities to screen the influence of these ionized impurities, thereby extending the carrier lifetime and reducing the bound state linewidth.

We have demonstrated the possibility of utilizing the shutoff region of resonant tunneling as an alternative and superior spectroscopic tool for measuring the intrinsic linewidth of electronic bound state in heterostructure systems, for a range of device temperatures. Future iterations of this work would make use of similar thick-barrier structures in the absence of the spacecharge region to reduce complications arising in the band profile of the device.

See supplementary material for a complete study of the modulation broadening amplitude on the resonant tunneling signature.

\section{Acknowledgments}

The authors would like to acknowledge Prof. Bill Frensley for the use of the numerical heterostructure band structure modeling program ('Bandprof").

Funding: This research was supported by the National Science Foundation [grant number 1309898].

[1] M. A. Reed, J. N. Randall, and J. H. Luscombe, Semiconductor Science and Technology 7, B12 (1992)

[2] M. Tewordt, L. Martin-Moreno, V. J. Law, M. J. Kelly, R. Newbury, M. Pepper, D. A. Ritchie, J. E. F. Frost, and G. A. C. Jones, Physical Review B 46, 3948 (1992)

[3] J. P. Eisenstein, L. N. Pfeiffer, and K. W. West, Applied Physics Letters 58, 1497 (1991)

[4] J. P. Eisenstein, Superlattices and Microstructures 12, 8 (1992)

[5] A. K. Geim, P. C. Main, N. La Scala, L. Eaves, T. J. Foster, P. H. Beton, J. W. Sakai, F. W. Sheard, M. Henini, G. Hill, and M. A. Pate, Phys Rev Lett 72, 2061 (1994)

[6] M. Tsuchiya and H. Sakaki, Japanese Journal of Applied Physics 30, 1164 (1991)

[7] Z. J. Qiu, Y. S. Gui, S. L. Guo, N. Dai, J. H. Chu, X. X. Zhang, and Y. P. Zeng, Applied Physics Letters 84, 1961 (2004)

[8] T. Oobo, R. Takemura, K. Sato, M. Suhara, Y. Miyamoto, and K. Furuya, Japanese Journal of Applied Physics 37, 445 (1998)

[9] Y. C. Kang, M. Suhara, K. Furuya, M. Gault, and R. Takemura, Physica B 227, 210 (1996)

[10] K. J. P. Jacobs, B. J. Stevens, and R. A. Hogg, IEICE Transactions on Electronics E99.C, 181 (2016)

[11] M. A. Reed, W. R. Frensley, W. M. Duncan, R. J. Matyi, A. C. Seabaugh, and H. L. Tsai, Applied Physics Letters 54, 1256 (1989)

[12] B. Ricco and M. Y. Azbel, Physical Review B 29, 1970 (1984)

[13] R. Tsu and L. Esaki, Applied Physics Letters 22, 562 (1973) 
[14] C. B. Duke, Tunneling in solids, Solid state physics Supplement (Academic Press, New York, 1969) p. 353 p.

[15] P. H. Rivera and P. A. Schulz, Brazilian Journal of Physics 26 (1996).

[16] N. Turner, J. Nicholls, E. Linfield, K. Brown, G. Jones, and D. Ritchie, Phys Rev B 54, 11 (1996)

[17] A. Gold, Physical Review B 38, 10798 (1988) 


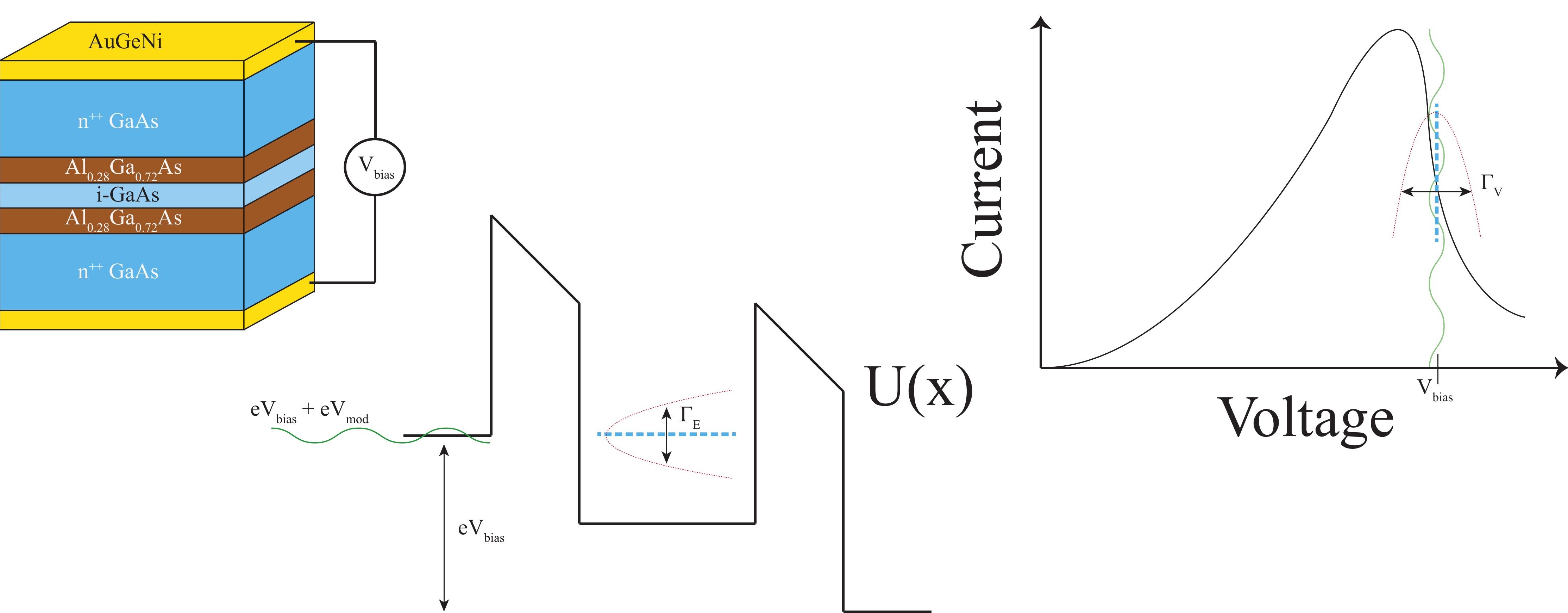

